\title{
Synthesis, Spectral Characterization and Anticonvulsant Studies of the Novel Triazolothiadiazoles Bearing Benzoxazole Moiety
}

\author{
MOHAMMAD SARAFROZ ${ }^{1}$, YASMIN KHATOON ${ }^{2 *}$, MOHD AMIR ${ }^{3}$, SALAHUDDIN $^{4}$, \\ MOHAMAD TALEUZZAMAN ${ }^{5}$ and CHANDAN YADAV ${ }^{6}$
}

\author{
'Department of Pharmaceutical Chemistry, College of Clinical Pharmacy, Imam Abdulrahman Bin \\ Faisal University, P.O. Box 1982, City Dammam, Saudi Arabia. \\ ${ }^{2,6}$ Department of Pharmacy, Jahangirabad Institute of Technology, Barabanki, Uttar Pradesh, India. \\ ${ }^{3}$ Department of Natural Product and Alternative Medicines, College of Clinical Pharmacy, \\ Imam Abdulrahman Bin Faisal University, P.O. Box 1982, City Dammam, Saudi Arabia. \\ ${ }^{4}$ Department of Pharmaceutical Chemistry, Noida institute of Engineering and Technology \\ (Pharmacy Institute), Greater Noida, Uttar Pradesh, India. \\ ${ }^{5}$ Department of Pharmaceutical Chemistry, Faculty of Pharmacy, Maulana Azad University, \\ Jodhpur, Rajasthan 342003, India. \\ ${ }^{*}$ Corresponding author E-mail: yassu.ayaan@gmail.com \\ http://dx.doi.org/10.13005/ojc/370408
}

(Received: April 07, 2021; Accepted: August 04, 2021)

\begin{abstract}
In this study, new fused triazolo-thiadiazoles $\left(4^{a-0}\right)$ were synthesized via methyl 2-[bromo(phenyl)methyl]-1,3-benzoxazole-5-carboxylate. The structure of novel derivatives was recognized on the basis of spectral data results and screened their anticonvulsant action by means of maximal electroshock seizure (MES) and subcutaneous pentylenetetrazol (scPTZ) procedures. Minimal motor studies were completed by a rotarod method. Compounds $4 \mathrm{e}, 4 \mathrm{~g}, 4 \mathrm{j}, 4 \mathrm{l}, 4 \mathrm{~m}$ and $4 \mathrm{n}$ showing better anticonvulsant action corresponding to hydrophobicity. Other molecules remained fewer lipophilic and have less effectiveness. Most of the compounds positively tolerable the rotarod test deprived of motor deficiency. In conclusion, the prepared derivatives with distal aryl moiety exhibited higher lipophilic character and lead to improved pharmacological achievement, which can be a forthcoming promise.
\end{abstract}

Keywords: Benzoxazole, Triazolothiadiazoles, Anticonvulsant, Neurotoxicity.

\section{INTRODUCTION}

Epilepsy is a disorder of neurological ailment portrayed by the occasional, unusual and hypersynchronous release of numbers of neurons that influence persons of all ages ${ }^{1-2}$. It is the acts of abrupt qualitative and quantitative unsettling influences of consciousness, and of sensor, autonomic and motor function and is the second most predominant neurological scatter in the industrial world ${ }^{3}$. In worldwide battle, World Health Organization (WHO) targeting epilepsy by

This is an Open Access article licensed under a Creative Commons license: Attribution 4.0 International (CC- BY). Published by Oriental Scientific Publishing Company @ 2018

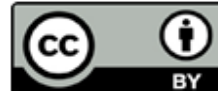


the organization with the International Bureau for Epilepsy (IBE) plus International League Against Epilepsy (ILAE) roughly about $0.5-1 \%$ to the general populace will be affected, whenever is harrowed with this neurological disorder ${ }^{4}$. Each year nearly 2.5 million new cases are added to these numbers ${ }^{4-7}$. Some of the at present accessible dynamic medications, (for example, stiripentol, pregabalin, tiagabine, zonisamide, lamotrigine, topiramate, levetiracetam) have demonstrated to be convincing in diminishing seizure, whilst their therapeutic viability is overwhelmed by some negative side effects like nausea, hepatotoxicity, ataxia, anxiety, anorexia, drowsiness, hirsutism and gastrointestinal ailments lessen their therapeutic efficacy since they have not been directly connected with a particular receptor inside the brain ${ }^{8}$. It is too hard to even think about identifying regular pharmacophore liable for anticipation or capture of seizure action mostly on account of the variety of organic molecules and mode of action in regulating the seizures ${ }^{4}$. In addition, about $30 \%$ of patients have uncontrolled seizures ${ }^{8,9}$. Hence, the quest for antiepileptic compounds with a progressively particular action and least toxicity keeps on being a region of investigation in medicinal chemistry ${ }^{10,11}$. In any investigation three strategies are utilized for this purpose ${ }^{12}$, firstscreening of recently synthesized chemicals with various structure and obscure system of activity; second-structure varieties of notable antiepileptic drugs; third-discerning medication plan with the advancement of medications that are specifically combined for following up on track epileptogenic component ${ }^{13,14}$. From the past investigations it was indicated that anticonvulsant activities have been appeared by various triazoles and thiadiazoles. The prime necessity was to look through the molecule that could have a combination of both the structures. This time initiates the synthesis of the fused triazolothiadiazole core. Literature study exposes that no attentive idea for antiepileptic action of the fused triazolo-thiadiazoles.

Thus, that was supposed to prepare and assimilate a fusion of 1,2,4-triazolo-1,3,4-thiadiazoles as the basic nucleus got together with substituted benzoxazole moiety within a solitary casing. This kind of assemblage is lead to better lipophilic nature with favorable anticonvulsant properties. Fused derivatives of triazolo-thiadiazole had been located for miscellaneous biological action take the instance of, anti-inflammatory ${ }^{15-17}$, antimicrobial ${ }^{18,19}$ and anticancer ${ }^{20,21}$. Substituted triazolo-thiadiazoles is an instance of the heterocyclic compound of different biological behavior is seen as one of the novel modules of anticonvulsants as discovered in a review of literatures ${ }^{22}$. Our point was to check for a potential anticonvulsant action of the recently synthetized fused triazolo-thiadiazole nucleus.

\section{MATERIALS AND METHODS}

\section{Chemistry}

All the substances and solvents employed throughout synthesis provided from CDS, SD Fine chemicals (India) and Merck. On a digital melting point apparatus, the melting point was resolved and is uncorrected. All the chemical reactions were checked by TLC performed on aluminium sheets precoated with silica gel G (Merck, India) by using the appropriate solvent systems. By using, (BIO-RAD FTS), FT-IR spectrophotometer the IR spectra are registered via $\mathrm{KBr}$ optics. ${ }^{1} \mathrm{H}-\mathrm{NMR}$ spectra proved with DRX-300 NMR spectrometer and BRUKER 400 Ultra ShieldTM and chemical changes $(\delta)$ in ppm in DMSO $-\mathrm{d}_{6} / \mathrm{CDCl}_{3}$ comparative to tetramethylsilane (TMS). The spectra of mass were found through the UPLC-MS/MS spectrometer (WATERS, version 4. 1 of Mass Lynx). Utilizing Perkin-Elmer model 240 analyzer for elemental analysis and all investigation were reliable within $\pm 0.4 \%$. The spots of TLC visualized under UV-light and lodine chamber.

\section{Synthesis \\ Synthesis of benzoxazole carboxylate}

Acombination of a-bromo phenylacetic acid and methyl-3-amino-4-hydroxybenzoate $(0.01 \mathrm{~mol})$ refluxed about 15 hours. Then reaction mixtures poured, cooled over the squashed ice by mixing for acquire the compounds $\mathrm{s}^{8,10}$.

\section{Synthesis of compound (1)}

In absolute ethanol, an equimolar quantity of a mixture of hydrazine hydrate and methyl 2-[bromo(phenyl)methyl]-1,3-benzoxazole-5carboxylate was heated under the reflux (18-20 hours). The obtained compound was cooled, the solid precipitate was filtered and recrystallized by using ethanol ${ }^{8,10}$.

\section{Synthesis of compound (2)}

An equimolar number of compound (1) and 
carbon disulfide were stirred at room temperature for 10-12 $\mathrm{h}$ in presence of $25 \mathrm{~mL}$ alcoholic potassium hydroxide solution. The resulting mixture were cooled and condensed to ice. Separated potassium dithiocarbazinate washed with ether, and dried ${ }^{22}$.

\section{Synthesis of compound (3)}

The hydrazine hydrate $(0.04 \mathrm{~mol})$ and compound (2) $(0.02 \mathrm{~mol})$ in water $(50 \mathrm{~mL})$ remained refluxed for about $10-15 \mathrm{~h}$ through random pulsation. The shade to the resulting blend altered into light green per advancement from hydrogen sulfide gas. A consistent combination acquired during the progression of reaction. The resulting mixture cooled to normal temperature then diluted by using cold water $(20 \mathrm{~mL})$. Upon acidification with dil. $\mathrm{HCl}$, a whitish precipitate was obtained, which was filtered and rinsed with cooled water and dried ${ }^{22}$.

\section{General method used to prepare compounds (4a-0)}

In a combination of substituted aromatic acids and compound (3) (0.01 mol) phosphorous oxychloride $(10 \mathrm{~mL})$ was applied and refluxed for nearly five hours. Once the reaction was completed it was poured drop by drop into the chilled water, alkalified with aq. ammonia, which was filtered and crystallized with ethanol to achieve compound (4a). The other compounds (4b-o) have been synthesized by the same methods ${ }^{22}$.

\section{Compound (4a)}

IR $\left(\mathrm{KBr}, \mathrm{cm}^{-1}\right): 3001$ (C-H aromatic), 1609 $(\mathrm{C}=\mathrm{N}), 1478(\mathrm{C}-\mathrm{N}), 1227(\mathrm{~N}-\mathrm{N}=\mathrm{C}), 711$ (C-S-C), 522 (C-Br). ${ }^{1} \mathrm{H}-\mathrm{NMR}$ (DMSO- $\left.{ }_{\mathrm{d} 6}, \delta, \mathrm{ppm}\right):$ 7.51-8.17 $(13 \mathrm{H}, \mathrm{m}, \mathrm{Ar}-\mathrm{H}), 6.33(1 \mathrm{H}, \mathrm{s}, \mathrm{CHBr})$. Anal calc. for $\mathrm{C}_{23} \mathrm{H}_{14} \mathrm{BrN}_{5} \mathrm{OS}$ : C, 56.78; $\mathrm{H}, 3.021 ; \mathrm{N}, 14.18$. Found: C, 56.57; H, 2.85; N, 14.34 .

\section{Compound (4b)}

IR $\left(\mathrm{KBr}, \mathrm{cm}^{-1}\right): 3008$ (C-H aromatic), 1611 $(\mathrm{C}=\mathrm{N}), 1471(\mathrm{C}-\mathrm{N}), 1234(\mathrm{~N}-\mathrm{N}=\mathrm{C}), 705$ (C-S-C), 525 (C-Br). ${ }^{1} \mathrm{H}-\mathrm{NMR}$ (DMSO- $\left.{ }_{\mathrm{d} 6}, \delta, \mathrm{ppm}\right): 7.22-8.09(12 \mathrm{H}$, $\mathrm{m}, \mathrm{Ar}-\mathrm{H}), 6.33(1 \mathrm{H}, \mathrm{s}, \mathrm{CHBr}), 2.86\left(3 \mathrm{H}, \mathrm{s}, \mathrm{CH}_{3}\right)$. Anal. calc. for $\mathrm{C}_{24} \mathrm{H}_{16} \mathrm{BrN}_{5} \mathrm{OS}$ : C, 56.99; H, 3.12; N, 13.62 . Found: C, 57.38; H, 3.21; N, 13.94 .

\section{Compound (4c)}

IR (KBr, cm$\left.{ }^{-1}\right): 3034$ (C-H aromatic), 1601 $(\mathrm{C}=\mathrm{N}), 1499$ (C-N), 1225 (N-N=C), 710 (C-S-C), 529 (C-Br). ${ }^{1} \mathrm{H}-\mathrm{NMR}\left(\mathrm{DMSO}_{\mathrm{d} 6}, \mathrm{\delta}, \mathrm{ppm}\right):$ 7.19-8.11 (12H, m, Ar-H), $6.27(1 \mathrm{H}, \mathrm{s}, \mathrm{CHBr}), 2.57\left(3 \mathrm{H}, \mathrm{s}, \mathrm{CH}_{3}\right)$. Anal. Calc. for $\mathrm{C}_{24} \mathrm{H}_{16} \mathrm{BrN}_{5} \mathrm{OS}$ : C, 56.99; $\mathrm{H}, 3.12 ; \mathrm{N}, 13.62$. Found: C, 57.38; H, 3.21; N, 13.94 .

\section{Compound (4d)}

IR $\left(\mathrm{KBr}, \mathrm{cm}^{-1}\right): 3022$ (C-H aromatic), 1613 $(\mathrm{C}=\mathrm{N}), 1482(\mathrm{C}-\mathrm{N}), 1221(\mathrm{~N}-\mathrm{N}=\mathrm{C}), 717$ (C-S-C), 545 (C-Br). ${ }^{1} \mathrm{H}-\mathrm{NMR}\left(\mathrm{DMSO}{ }_{\mathrm{d} 6}, \delta, \mathrm{ppm}\right): 7.26-8.14(12 \mathrm{H}$, $\mathrm{m}, \mathrm{Ar}-\mathrm{H}), 6.34(1 \mathrm{H}, \mathrm{s}, \mathrm{CHBr}), 2.46\left(3 \mathrm{H}, \mathrm{s}, \mathrm{CH}_{3}\right)$. Anal. calc. for $\mathrm{C}_{24} \mathrm{H}_{16} \mathrm{BrN}_{5} \mathrm{OS}$ : C, 56.99; $\mathrm{H}, 3.12 ; \mathrm{N}, 13.62$. Found: $\mathrm{C}, 57.38 ; \mathrm{H}, 3.21 ; \mathrm{N}, 13.94$.

\section{Compound (4e)}

IR (KBr, cm-1): 3078 (C-H aromatic), 1622 $(\mathrm{C}=\mathrm{N}), 1512(\mathrm{C}-\mathrm{N}), 1201(\mathrm{~N}-\mathrm{N}=\mathrm{C}), 711$ (C-S-C). 544 (C-Br). ${ }^{1} \mathrm{H}-\mathrm{NMR}$ (DMSO- $\left.{ }_{\mathrm{d} 6}, \delta, \mathrm{ppm}\right):$ 7.27-8.21 $(12 \mathrm{H}, \mathrm{m}, \mathrm{Ar}-\mathrm{H}), 6.56(1 \mathrm{H}, \mathrm{s}, \mathrm{CHBr}), 4.56(2 \mathrm{H}, \mathrm{s}$, $\left.\mathrm{CH}_{2} \mathrm{Br}\right)$. Mass m/z: $579.940(\mathrm{M}+1)$. Anal. calc. for $\mathrm{C}_{24} \mathrm{H}_{15} \mathrm{Br}_{2} \mathrm{~N}_{5} \mathrm{OS}$ : C, 49.79; $\mathrm{H}, 2.44 ; \mathrm{N}, 12.36$. Found: C, 49.59; H, 2.60; N, 12.05.

\section{Compound (4f)}

IR $\left(\mathrm{KBr}, \mathrm{cm}^{-1}\right): 3033$ (C-H aromatic), 1611 $(\mathrm{C}=\mathrm{N}), 1480$ (C-N), 1227 (N-N=C), 711 (C-S-C), 522 (C-Br). ${ }^{1} \mathrm{H}-\mathrm{NMR}$ (DMSO- $\left.{ }_{\mathrm{d} 6}, \delta, \mathrm{ppm}\right)$ : 7.03-8.11 (12H, m, Ar-H), $6.32(1 \mathrm{H}, \mathrm{s}, \mathrm{CHBr}), 3.86\left(3 \mathrm{H}, \mathrm{s}, \mathrm{OCH}_{3}\right)$. Anal. calc. for $\mathrm{C}_{24} \mathrm{H}_{16} \mathrm{BrN}_{5} \mathrm{O}_{2} \mathrm{~S}: \mathrm{C}, 55.83 ; \mathrm{H}, 3.01 ; \mathrm{N}$, 13.48. Found: C, 55.61; H, 3.11; N, 13.51 .

\section{Compound (4g)}

IR $\left(\mathrm{KBr}, \mathrm{cm}^{-1}\right): 3036$ (C-H aromatic), 1591 $(\mathrm{C}=\mathrm{N}), 1478(\mathrm{C}-\mathrm{N}), 1239(\mathrm{~N}-\mathrm{N}=\mathrm{C}), 717$ (C-S-C), 523 (C-Br), ${ }^{1} \mathrm{H}-\mathrm{NMR}$ (DMSO- $\left.{ }_{\mathrm{d} 6}, \delta, \mathrm{ppm}\right):$ 6.93-8.09 $(13 \mathrm{H}, \mathrm{m}, \mathrm{Ar}-\mathrm{H}), 6.22(1 \mathrm{H}, \mathrm{s}, \mathrm{CHBr}) .5 .06(2 \mathrm{H}, \mathrm{s}$, $\left.\mathrm{OCH}_{2}\right)$. Mass m/z: $518.029(\mathrm{M}+1)$. Anal. calc. for $\mathrm{C}_{24} \mathrm{H}_{16} \mathrm{BrN}_{5} \mathrm{O}_{2} \mathrm{~S}: \mathrm{C}, 55.85 ; \mathrm{H}, 3.43 ; \mathrm{N}, 13.67$. Found: C, 55.61; H, 3.11; N, 13.51 .

\section{Compound (4h)}

IR $\left(\mathrm{KBr}, \mathrm{cm}^{-1}\right): 3065$ (C-H aromatic), 1733 $(\mathrm{C}=\mathrm{O}), 1611(\mathrm{C}=\mathrm{N}), 1489(\mathrm{C}-\mathrm{N}), 1223(\mathrm{~N}-\mathrm{N}=\mathrm{C})$, 711 (C-S-C), 520 (C-Br). ${ }^{1} \mathrm{H}-\mathrm{NMR}$ (DMSO- ${ }_{\mathrm{d} 6}$, $\delta$, ppm): 10.11 (1H, s, CHO), 7.12-8.08 (12H, m, Ar-H), $6.32(1 \mathrm{H}, \mathrm{s}, \mathrm{CHBr}), 5.24\left(2 \mathrm{H}, \mathrm{s}, \mathrm{OCH}_{2}\right)$. Mass m/z: $546.022(\mathrm{M}+1)$. Anal. calc. for $\mathrm{C}_{25} \mathrm{H}_{16} \mathrm{BrN}_{5} \mathrm{O}_{3} \mathrm{~S}: \mathrm{C}$, 54.44; H, 3.08; N, 12.99. Found: C, 54.95; H, 2.95; $\mathrm{N}, 12.82$.

\section{Compound (4i)}

IR $\left(\mathrm{KBr}, \mathrm{cm}^{-1}\right): 3033$ (C-H aromatic), 1738 $(\mathrm{C}=\mathrm{O}), 1623(\mathrm{C}=\mathrm{N}), 1490(\mathrm{C}-\mathrm{N}), 1223(\mathrm{~N}-\mathrm{N}=\mathrm{C}), 692$ 
(C-S-C), 524 (C-Br). ${ }^{1} \mathrm{H}-\mathrm{NMR}$ (DMSO- $\left.{ }_{\mathrm{d} 6}, \delta, \mathrm{ppm}\right)$ : $10.03(1 \mathrm{H}, \mathrm{s}, \mathrm{CHO}), 7.36-8.08(12 \mathrm{H}, \mathrm{m}, \mathrm{Ar}-\mathrm{H}), 6.44$ $(1 \mathrm{H}, \mathrm{s}, \mathrm{CHBr})$. Mass $\mathrm{m} / \mathrm{z}$ : $516.012(\mathrm{M}+1)$. Anal. calc. for $\mathrm{C}_{24} \mathrm{H}_{14} \mathrm{BrN}_{5} \mathrm{O}_{2} \mathrm{~S}: \mathrm{C}, 55.41 ; \mathrm{H}, 2.38 ; \mathrm{N}, 13.25$. Found: C, 55.82; $\mathrm{H}, 2.73 ; \mathrm{N}, 13.56$.

\section{Compound (4j)}

IR $\left(\mathrm{KBr}, \mathrm{cm}^{-1}\right): 3020$ (C-H aromatic), 1611 $(\mathrm{C}=\mathrm{N}), 1498(\mathrm{C}-\mathrm{N}), 1256(\mathrm{~N}-\mathrm{N}=\mathrm{C}), 698(\mathrm{C}-\mathrm{S}-\mathrm{C})$, 557 (C-Br). ${ }^{1} \mathrm{H}-\mathrm{NMR}$ (DMSO- $\left.{ }_{\mathrm{d} 6}, \delta, \mathrm{ppm}\right): 9.26(1 \mathrm{H}$, $\mathrm{s}$, pyridine), 7.48-8.79 $(11 \mathrm{H}, \mathrm{m}, \mathrm{Ar}-\mathrm{H}), 6.55(1 \mathrm{H}, \mathrm{s}$, CHBr). Anal. calc. for $\mathrm{C}_{22} \mathrm{H}_{13} \mathrm{BrN}_{6} \mathrm{OS}$ : C, 54.13; $\mathrm{H}$, 3.01 ; N, 17.32. Found: C, 54.00; H, 2.68; N, 17.17.

\section{Compound (4k)}

IR $\left(\mathrm{KBr}, \mathrm{cm}^{-1}\right): 3037$ (C-H aromatic), 1601 $(\mathrm{C}=\mathrm{N}), 1484(\mathrm{C}-\mathrm{N}), 1244,(\mathrm{~N}-\mathrm{N}=\mathrm{C}), 700$ (C-S-C), 514 (C-Br). ${ }^{1} \mathrm{H}-\mathrm{NMR}$ (DMSO- $\left.{ }_{\mathrm{d} 6}, \delta, \mathrm{ppm}\right): 7.21-8.14(13 \mathrm{H}$, $\mathrm{m}, \mathrm{Ar}-\mathrm{H}), 6.11(1 \mathrm{H}, \mathrm{s}, \mathrm{CHBr}), 3.77\left(2 \mathrm{H}, \mathrm{s}, \mathrm{CH}_{2}\right)$. Anal. calc. for $\mathrm{C}_{24} \mathrm{H}_{16} \mathrm{BrN}_{5} \mathrm{OS}$ : C, 57.10; $\mathrm{H}, 3.15 ; \mathrm{N}, 13.80$. Found: C, 57.38; H, 3.21; N, 13.94.

\section{Compound (4I)}

IR $\left(\mathrm{KBr}, \mathrm{cm}^{-1}\right): 3061$ (C-H aromatic), 1636 $(\mathrm{C}=\mathrm{N}), 1491(\mathrm{C}-\mathrm{N}), 1241(\mathrm{~N}-\mathrm{N}=\mathrm{C}), 733(\mathrm{C}-\mathrm{Cl}), 711$ (C-S-C), 533 (C-Br). ${ }^{1} \mathrm{H}-\mathrm{NMR}$ (DMSO- $\left.{ }_{\mathrm{d} 6}, \delta, \mathrm{ppm}\right)$ : 7.16-8.11 (12H, m, Ar-H), 6.46 (1H, s, CHBr), 2.81 $\left(2 \mathrm{H}, \mathrm{s}, \mathrm{CH}_{2}\right)$. Anal. calc. for $\mathrm{C}_{24} \mathrm{H}_{15} \mathrm{BrClN}_{5} \mathrm{OS}$ : C, 53.65; $\mathrm{H}, 3.01 ; \mathrm{N}, 13.44$. Found: $\mathrm{C}, 53.70 ; \mathrm{H}, 2.82 ; \mathrm{N}, 13.05$.

\section{Compound (4m)}

IR ( $\left.\mathrm{KBr}, \mathrm{cm}^{-1}\right): 3075$ (C-H aromatic), 1612 $(\mathrm{C}=\mathrm{N}), 1512(\mathrm{C}-\mathrm{N}), 1255(\mathrm{~N}-\mathrm{N}=\mathrm{C}), 710$ (C-S-C), 566 (C-Br). ${ }^{1} \mathrm{H}-\mathrm{NMR}$ (DMSO- $\left.{ }_{\mathrm{d} 6}, \delta, \mathrm{ppm}\right): 7.03-8.08(12 \mathrm{H}$, $\mathrm{m}, \mathrm{Ar}-\mathrm{H}), 6.21(1 \mathrm{H}, \mathrm{s}, \mathrm{CHBr}), 3.77\left(2 \mathrm{H}, \mathrm{s}, \mathrm{CH}_{2}\right)$. Anal. calc. for $\mathrm{C}_{24} \mathrm{H}_{15} \mathrm{Br}_{2} \mathrm{~N}_{5} \mathrm{OS}$ : C, 49.11; $\mathrm{H}, 2.13 ; \mathrm{N}, 12.40$. Found: C, 49.59; H, 2.60; N, 12.05.

\section{Compound (4n)}

IR $\left(\mathrm{KBr}, \mathrm{cm}^{-1}\right): 3048$ (C-H aromatic), 1633 $(\mathrm{C}=\mathrm{N}), 1510(\mathrm{C}-\mathrm{N}), 1234(\mathrm{~N}-\mathrm{N}=\mathrm{C}), 711$ (C-S-C), 531 (C-Br). ${ }^{1} \mathrm{H}-\mathrm{NMR}$ (DMSO- $\left.{ }_{\mathrm{d} 6}, \delta, \mathrm{ppm}\right):$ 7.21-8.21 $(13 \mathrm{H}, \mathrm{m}, \mathrm{Ar}-\mathrm{H}), 6.36(2 \mathrm{H}, \mathrm{s}, 2 \times \mathrm{CHBr})$. Anal. calc. for $\mathrm{C}_{24} \mathrm{H}_{15} \mathrm{Br}_{2} \mathrm{~N}_{5} \mathrm{OS}$ : C, 50.00; $\mathrm{H}, 2.13 ; \mathrm{N}, 12.32$. Found: C, 49.59; H, 2.60; N, 12.05 .

\section{Compound (40)}

IR $\left(\mathrm{KBr}, \mathrm{cm}^{-1}\right): 3576(\mathrm{OH}), 2998(\mathrm{C}-\mathrm{H}$ aromatic), $1608(\mathrm{C}=\mathrm{N}), 1490(\mathrm{C}-\mathrm{N}), 1235(\mathrm{~N}-\mathrm{N}=\mathrm{C})$, 701 (C-S-C), 534 (C-Br). ${ }^{~ H} \mathrm{H}-\mathrm{NMR}$ (DMSO- $\left.{ }_{\mathrm{d} 6}, \delta, \mathrm{ppm}\right)$ : $10.78(3 \mathrm{H}, \mathrm{s}, 3 \times \mathrm{OH}), 6.86-8.98(1 \mathrm{HH}, \mathrm{m}, \mathrm{Ar}-\mathrm{H}), 6.33$
$(1 \mathrm{H}, \mathrm{s}, \mathrm{CHBr})$. Anal. calc. for $\mathrm{C}_{23} \mathrm{H}_{14} \mathrm{BrN}_{5} \mathrm{O}_{4} \mathrm{~S}$ : C, 51.34; $\mathrm{H}, 2.44 ; \mathrm{N}, 14.83$. Found: C, 51.50; H, 2.63; N, 14.90 .

\section{Biological Activity Anticonvulsant Screening}

Each of those compounds were tested using SCPTZ and MES methods for their antiepileptic behavior, as demonstrated by the National Institute of Anticonvulsant Screening Program²3-24.

Compounds were suspended in polyethylene glycol and tested by the Institutional Animal Ethics Committee, R.V. Northland College, Dadri, Greater Noida, Uttar Pradesh, India, under the RVNI/IAEC/2017/05 proposal number. Finding rata are listed in Table 2.

\section{Maximum Electroshock (MES)Test}

Mice received a $50-\mathrm{mA} 60 \mathrm{~Hz}$ ac voltage for $0.2 \mathrm{~s}$ via ear pins, and the responses were analyzed at 0.5 and $4.0 \mathrm{~h}$ after an i.p. injection of the product $(30,100$, and $300 \mathrm{mg} / \mathrm{kg})$. Consequently, protection against seizure was noted ${ }^{22}$.

\section{Pentylenetetrazole Test}

Intra peritoneal at $75 \mathrm{mg} / \mathrm{kg} \mathrm{scPTZ}$ was injected which produces seizures in the mice. The dose of test analogs was found to the animals for 30 min later. Defense against clonic spasm was described as protection against the spread of seizures of at least $5 \mathrm{~s}$ duration ${ }^{22,23}$.

\section{Neurotoxicity test}

The neurological intervention was shown on the mice using rotarod method ${ }^{21,22}$. The tested mice failure to manage equilibrium during at least one minute for two successive studies on a continuously circulating plastic rod $(10 \mathrm{rpm})$ with a diameter of $2.3 \mathrm{~cm}$ proposed motor dysfunction.

\section{Lipophilic/Hydrophobic identification}

Lipophilic character regulates the efficacy of synthesized compounds working on the brain, determined by established procedure ${ }^{25}$. It was found that the highest efficacy of the compounds acting in the central nervous system is achieved by congeners possessing ideal lipophilicity $(\log P)$ close to two and was determined using chloroform phosphate with the measured $\log P$ value ${ }^{26}$. 


\section{RESULTS AND DISCUSSION}

Scheme 1 details the synthetic route used to synthesize title compounds. Methyl-2-[bromo(phenyl) methyl]-1,3-benzoxazole-5-carboxylate and its hydrazide was produced by the reaction of methyl4-hydroxy benzoate and a mixture of aluminum nitrate, acetic anhydride, 2-bromo-2-phenylacetic acid and hydrazine hydrate ${ }^{4}$. In the presence of potassium hydroxide, the acid hydrazide (1) reacted with carbon disulfide to provide potassium 2-[2-(3-chlorophenyl)-1,3- benzoxazol-5-yl] carbonyl hydrazine dithiocarbonate (2). This salt was cyclized to give 4-amino-5-[2-(3-chlorophenyl)- 1,3-benzoxazol5-yl\}-4H-1,2,4-triazole-3-thiol (3) by an excess of hydrazine hydrate. In the presence of phosphorous oxychloride, the resulting triazole was further converted to titled compounds by condensing with specific aromatic acids by one-pot reaction. In all cases the product's TLC revealed single spot that verified the chromatogram. The physical constants record in Table 1. Structure (3) was verified through results from ${ }^{1} \mathrm{H}-\mathrm{NMR}$, displayed a singlet downfield attributed to the $-\mathrm{SH}$ group at around $13.85 \mathrm{ppm}$ and amino group seemed as a singlet at about 5.63ppm, respectively. Lack of peak of amino and -SH indicates, triazole was changed into titled compounds (4a-n) by retorting with the aromatic acid moiety $-\mathrm{COOH}$. The structure of prepared compounds distinguished through elemental analysis and spectral data information were noted to experimental protocols.

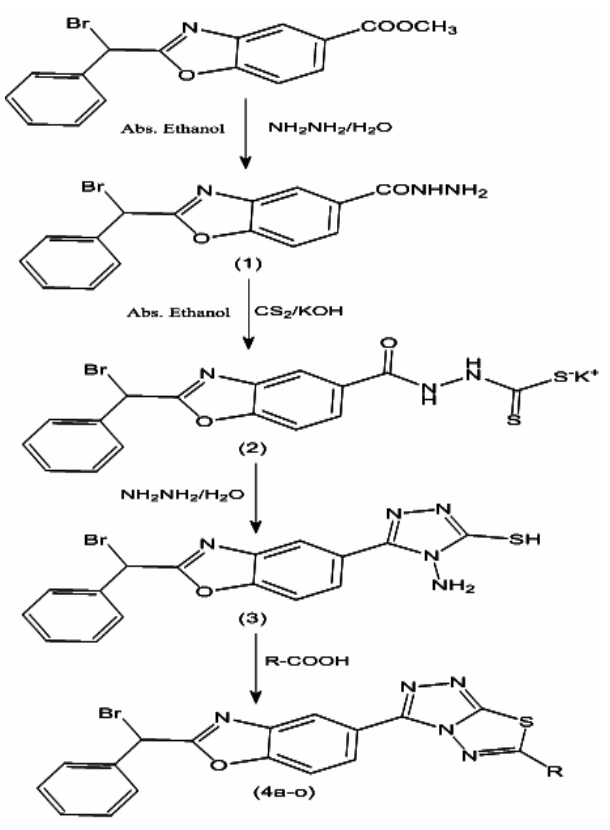

Scheme 1. Synthesis of fused triazolothiadiazoles

Table 1: Physical constants of the synthesized molecules (4a-0)

\begin{tabular}{|c|c|c|c|c|c|c|}
\hline S.No. & $\mathrm{R}$ & Mol. Formula & ${ }^{\mathrm{b}} \mathrm{M} . \mathrm{P}\left({ }^{\circ} \mathrm{C}\right)$ & ${ }^{\circ} \log P$ & Yield(\%) & ${ }^{\mathrm{d}} \mathrm{Rf}$ Value \\
\hline $4 a$ & $\mathrm{C}_{6} \mathrm{H} 5$ & $\mathrm{C}_{23} \mathrm{H}_{14} \mathrm{BrN}_{5} \mathrm{OS}$ & $130-132$ & 0.84 & 67 & 0.80 \\
\hline $4 b$ & $2-\mathrm{CH}_{3} \mathrm{C}_{6} \mathrm{H}_{4}$ & $\mathrm{C}_{24} \mathrm{H}_{16} \mathrm{BrN}_{5} \mathrm{OS}$ & $145-147$ & 0.84 & 67 & 0.80 \\
\hline $4 c$ & $3-\mathrm{CH}_{3} \mathrm{C}_{6} \mathrm{H}_{4}$ & $\mathrm{C}_{24} \mathrm{H}_{16} \mathrm{BrN}_{5} \mathrm{OS}$ & $150-152$ & 0.85 & 56 & 0.81 \\
\hline $4 d$ & $4-\mathrm{CH}_{3} \mathrm{C}_{6} \mathrm{H}_{4}$ & $\mathrm{C}_{24} \mathrm{H}_{16} \mathrm{BrN}_{5} \mathrm{OS}$ & $145-147$ & 0.99 & 55 & 0.61 \\
\hline $4 \mathrm{e}$ & 4- $\mathrm{BrCH}_{2} \mathrm{C}_{6} \mathrm{H}_{4}$ & $\mathrm{C}_{24} \mathrm{H}_{15} \mathrm{Br}_{2} \mathrm{~N}_{5} \mathrm{OS}$ & $160-152$ & 2.54 & 45 & 0.70 \\
\hline $4 f$ & $4-\mathrm{OCH}_{3} \mathrm{C}_{6} \mathrm{H}_{4}$ & $\mathrm{C}_{24} \mathrm{H}_{16} \mathrm{BrN}_{5} \mathrm{O}_{2} \mathrm{~S}$ & $150-152$ & 0.56 & 40 & 0.70 \\
\hline $4 g$ & $\mathrm{C}_{6} \mathrm{H}_{5} \mathrm{OCH}_{2}$ & $\mathrm{C}_{24} \mathrm{H}_{16} \mathrm{BrN}_{5} \mathrm{O}_{2} \mathrm{~S}$ & $155-156$ & 1.87 & 77 & 0.75 \\
\hline $4 \mathrm{~h}$ & $4-\mathrm{CHOC}_{6} \mathrm{H}_{4} \mathrm{OCH}_{2}$ & $\mathrm{C}_{25} \mathrm{H}_{16} \mathrm{BrN}_{5} \mathrm{O}_{3} \mathrm{~S}$ & $160-162$ & 1.11 & 45 & 0.80 \\
\hline $4 i$ & $4-\mathrm{CHOC}_{6} \mathrm{H}_{4}$ & $\mathrm{C}_{24} \mathrm{H}_{14} \mathrm{BrN}_{5} \mathrm{O}_{2} \mathrm{~S}$ & $150-152$ & 1.03 & 67 & 0.63 \\
\hline $4 j$ & $\mathrm{C}_{5} \mathrm{H}_{4} \mathrm{~N}$ & $\mathrm{C}_{22} \mathrm{H}_{13} \mathrm{BrN}_{6} \mathrm{OS}$ & $180-181$ & 2.32 & 66 & 0.60 \\
\hline $4 \mathrm{k}$ & $\mathrm{C}_{6} \mathrm{H}_{5} \mathrm{CH}_{2}$ & $\mathrm{C}_{24} \mathrm{H}_{16} \mathrm{BrN}_{5} \mathrm{OS}$ & $140-142$ & 0.94 & 70 & 0.82 \\
\hline 41 & 4- $\mathrm{ClC}_{6} \mathrm{H}_{4} \mathrm{CH}_{2}$ & $\mathrm{C}_{24} \mathrm{H}_{15} \mathrm{BrClN}_{5} \mathrm{OS}$ & $175-176$ & 2.02 & 71 & 0.81 \\
\hline $4 m$ & $4-\mathrm{BrC}_{6} \mathrm{H}_{4} \mathrm{CH}_{2}$ & $\mathrm{C}_{24} \mathrm{H}_{15} \mathrm{Br}_{2} \mathrm{~N}_{5} \mathrm{OS}$ & $155-157$ & 2.36 & 39 & 0.62 \\
\hline $4 n$ & $\mathrm{C}_{6} \mathrm{H}_{5} \mathrm{CHBr}$ & $\mathrm{C}_{24} \mathrm{H}_{15} \mathrm{Br}_{2} \mathrm{~N}_{5} \mathrm{OS}$ & $165-167$ & 2.41 & 45 & 0.51 \\
\hline 40 & 3,4,5-tri $\mathrm{OHC}_{6} \mathrm{H}_{2}$ & $\mathrm{C}_{23} \mathrm{H}_{14} \mathrm{BrN}_{5} \mathrm{O}_{4} \mathrm{~S}$ & $160-161$ & 1.14 & 70 & 0.66 \\
\hline
\end{tabular}

aSolvent of crystallization-ethanol.

${ }^{b}$ Molecules melted during the decomposition process.

'Log $\mathrm{P}$ was calculated at $30^{\circ} \mathrm{C}$ via chloroform/phosphate buffer method.

dSolvent system-benzene: acetone (8:2, v/v), benzene: ethanol (2:0.5, v/v), toluene: ethylacetate: formic acid (5: 4: $1, \mathrm{v} / \mathrm{v} / \mathrm{v})$.

Newly synthesized compounds (4a-o) were subjected to anticonvulsant activity, according to the standard protocol established by the division of epilepsy which requires the maximum electroshock seizure $^{8}$ and the subcutaneous pentylenetetrazole ${ }^{9}$.
In addition, the rotarod test can almost always detect acute toxicity of antiepileptic drugs in rodents ${ }^{11}$. Data are provided in Table 2. Standard drugs used for the analysis were phenytoin and carbamazepine. 
Table 2: Anticonvulsant and neurotoxicity studies of compounds (4a-o)

\begin{tabular}{ccccccc}
\hline $\begin{array}{c}\text { Compound } \\
(\mathrm{n}=4)\end{array}$ & \multicolumn{2}{c}{ (MES screen) } & \multicolumn{2}{c}{ i.p. injection in mice } & \multicolumn{2}{c}{ Neurotoxicity screening } \\
& $0.5 \mathrm{~h}$ & $4 \mathrm{~h}$ & $0.5 \mathrm{~h}$ & $4 \mathrm{~h}$ & $0.5 \mathrm{~h}$ & $4 \mathrm{~h}$ \\
\hline $4 \mathrm{a}$ & - & - & - & - & $\times$ & $\times$ \\
$4 \mathrm{~b}$ & 300 & - & - & - & - & 300 \\
$4 \mathrm{c}$ & 300 & - & - & - & $\times$ & $\times$ \\
$4 \mathrm{~d}$ & 300 & - & - & 300 & 300 & - \\
$4 \mathrm{e}$ & 30 & 300 & 300 & 300 & 300 & - \\
$4 \mathrm{f}$ & - & - & - & - & - & 300 \\
$4 \mathrm{~g}$ & 100 & 300 & 300 & - & 300 & - \\
$4 \mathrm{~h}$ & 300 & 300 & - & - & - & - \\
$4 \mathrm{i}$ & 300 & - & - & - & $\times$ & $\times$ \\
$4 \mathrm{j}$ & 30 & 300 & 300 & 300 & - & 300 \\
$4 \mathrm{k}$ & - & - & - & - & $\times$ & $\times$ \\
$4 \mathrm{l}$ & 100 & 300 & 300 & - & - & - \\
$4 \mathrm{~m}$ & 30 & 300 & 300 & - & - & 300 \\
$4 \mathrm{n}$ & 30 & 300 & 300 & - & 300 & - \\
40 & 300 & - & - & - & - & - \\
Phenytoin & 30 & 30 & - & - & 100 & 100 \\
Carbamazepine & 30 & 100 & 100 & 300 & 300 & 300 \\
\hline
\end{tabular}

aMice was given doses of 30,100 , and $300 \mathrm{mg} / \mathrm{kg}$ via intraperitoneal path. The table describes the average dose at which therapeutic efficacy were detected in half or more of the mice. The animals were detected at 0.5 and $4.0 \mathrm{~h}$ after obtaining the treatment. The dash (-) depicts the omission of effect at optimal dosage, while the

In the anticonvulsant screening, each compound except $4 \mathrm{a}, 4 \mathrm{f}$ and $4 \mathrm{k}$ suggested motivating intervention. Compounds $4 \mathrm{e}, 4 \mathrm{j}, 4 \mathrm{~m}$ and $4 \mathrm{n}$ believed to be positively competitive towards MES testing at $30 \mathrm{mg} / \mathrm{kg}$ dosage at interval of $0.5 \mathrm{~h}$ which is characteristic of its desire to stop seizing progression at a usually reduced dose. At a dose of $100 \mathrm{mg} /$ $\mathrm{kg}$ only two molecules ( $4 \mathrm{~g}$ and $4 \mathrm{l}$ ) demonstrated reasonable level of safety. The $4 \mathrm{e}, 4 \mathrm{~g}, 4 \mathrm{~h}, 4 \mathrm{j}, 4 \mathrm{l}, 4 \mathrm{~m}$ and $4 \mathrm{n}$ compounds showed activity at time intervals of both 0.5 and 4.0 hours. For this way, the most of the compounds showed an encouraging anticonvulsant effect at an interval of $0.5 \mathrm{~h}$ indicated they had an instant emergence but reduced duration of action.

In chemo shock test, compounds $4 \mathrm{e}, 4 \mathrm{~g}, 4 \mathrm{j}$, $4 \mathrm{l}, 4 \mathrm{~m}$ and $4 \mathrm{n}$ display behavior indicative of their ability to inhibit seizure spread at maximum dose during half an hour, signifying that fast onset but reduced duration of action. Only three molecules $4 d, 4 e$ and $4 \mathrm{j}$ became vigorous after prolonged operation duration of 4.0 hours. At both time intervals, only two compound $4 \mathrm{e}$ and $4 \mathrm{j}$ displayed action at maximum dose level.

Rotarod test used for the study of neurotoxicity to assessment undesired effects like sedation and ataxia induced through the compounds. Compounds $4 \mathrm{~h}, 4 \mathrm{l}$ and 40 showed no toxicity at the maximum doses. At 0.5 and $4.0 \mathrm{~h}$ no any molecules were toxic, while $4 \mathrm{~d}, 4 \mathrm{e}, 4 \mathrm{~g}$ and $4 \mathrm{n}$ showed toxicity at $0.5 \mathrm{~h}$ and no toxicity at 4.0 hours. Some 4b, 4f, $4 \mathrm{j}$ and $4 \mathrm{~m}$ compounds demonstrated delayed toxicity, i.e. toxicity after only $4.0 \mathrm{~h}$ which is equivalent to carbamazepine (300 mg/kg). Most of the compounds were however not as much of toxic than phenytoin $(100 \mathrm{mg} / \mathrm{kg})$.

Compounds $4 \mathrm{e}, 4 \mathrm{~g}, 4 \mathrm{j}, 4 \mathrm{I}, 4 \mathrm{~m}$ and $4 \mathrm{n}$ were found to have more lipophilic activity with active anticonvulsants. Other molecules were fewer lipophilic and were less active.

\section{CONCLUSION}

Fifteen new triazolothiadiazoles have been made and subjected for anticonvulsant movement by MES and scPTZ methods. All molecules exposed anticonvulsant action on MES screen except $4 \mathrm{a}, 4 \mathrm{f}$ and $4 \mathrm{k}$. The primary MES screening compounds $4 \mathrm{e}, 4 \mathrm{~g}, 4 \mathrm{~h}, 4 \mathrm{j}, 4 \mathrm{I}, 4 \mathrm{~m}$ and $4 \mathrm{n}$ displayed activity against seizures at both 0.5 and $4.0 \mathrm{~h}$, approving their potential value as per prototypic molecules. Anticonvulsant activity records exposed that most compounds exhibited notable reduction in the extender process of the hind limb tonic convulsion and molecules $4 \mathrm{e}, 4 \mathrm{j}, 4 \mathrm{~m}$ and $4 \mathrm{n}$ were to be the greatest 
effective. What's more, the anticonvulsant activities of different compounds contemplated have been seen as abundant less successful than standard drugs.

It appears that the existence of halo substituted aryl at benzoxazole moiety (such as chloro and bromo) and alkyl group attached to the triazolothiadiazole ring on the aryl ring showed the strongest anticonvulsant activity and favorable high safety. Some compounds such as $4 \mathrm{e}, 4 \mathrm{~g}, 4 \mathrm{j}, 4 \mathrm{l}, 4 \mathrm{~m}$ and $4 \mathrm{n}$ had high lipid character and were high active. Other candidates were also lipophilic, but were less active in MES screening. A significant lot of the formerly stated compounds have shown an advanced level of safety and could have a potential duty apparently.

\section{ACKNOWLEDGEMENT}

Authors are extremely indebted to IIT Delhi and Jamia Hamdard University for the spectral study of the synthesized molecules. We are also grateful for finalizing the assessment by adopted the Antiepileptic Drug Development Program.

\section{Conflict of interest}

No conflict of interest occurs between authors.

\section{REFERENCES}

1. Pottoo, F. H.; Tabassum, N.; Javed, M. N.; Nigar, S.; Rasheed, R.; Khan, A.; Barkat, M. A.; Alam, M. S.; Maqbool, A.; Ansari, M. A.; Barreto, G. E.; Ashraf, G. M. Mol. Neu., 2019, 56(2), 1233-1247.

2. Ahmad, N.; Ahmad, R.; Alrasheed, R. A.; Almatar, H. M. A.; Al-Ramadan, A. S.; Amir, M.; Sarafroz, M. Pharmaceu., 2020, 12, 1-34.

3. Nigar, S.; Pottoo, F. H.; Tabassum, N.; Verma, S. K.; Javed, M. N. J. Adv. Med. Pharm. Sci., 2016, 10, 1-9.

4. Khatoon, Y.; Singh, V.; Sarafroz, M. In. J. Pharm. Sci. Rev., 2018, 48(2), 70-78.

5. Ahmad, N.; Ahmad, R.; Al Qatifi, S.; Alessa, M.; Al Hajji, H.; Sarafroz, M. BMC Chem., 2020, 14(1), 1-15.

6. Łuszczki, J. J. Pharm. Rep., 2009, 61(2), 197-216.

7. Naveeda, R.; Anum, S.; Saima, K. In. J. App Sci. Res. Rev., 2018, 5, 2-10.

8. Siddiqui, N.; Sarafroz, M.; Alam, M. M.; Ahsan, W. Acta. Polo. Pharm. Drug Res., 2008, 4(65), 449-455.

9. Sarafroz, M.; Khatoon, Y.; Ahmad, N.; Amir, M.; Salahuddin.; Pottoo, F. H. Orient. J. Chem., 2019, 35(1), 64-70.

10. Remi, J.; Huttenbrenner, A.; Feddersen, B.; Noachtar, S. Epi. Res., 2010, 88, 145-150.

11. Sarafroz, M.; Khatoon, Y.; Ahmad, N.; Amir, M.; Salahuddin, Pottoo, F. H.; Taleuzzaman, M.; Ahmad, W. I. J. Pharm. Sci. Res., 2020, 11(1), 137-45.

12. Balestrini, S.; Sisodiya, S. M. Neu. Let., 2018, 22, 27-39.

13. Ali, M. R.; Marella, A.; Alam, T. M.; Naz, R.; Akhter, M.; Shaquiquzzaman, M.; Saha, R.; Tanwar, O.; Alam, M. M.; Hooda, J. Indo. J.
Pharm., 2012, 23(4), 193- 202.

14. Asif, M.; Husain, A. J. App. Chem., 2013, 66, 1-7.

15. El-Shehry, M. F.; Abu-Hashem, A. A.; ElTelbani, E. M. Eur. J. Med. Chem., 2010, 45, 1906-1911.

16. Amir, M.; Kumar, H.; Javed, S. A. Eur. J. Med. Chem., 2008, 43, 2056-2066.

17. Metwally, K. A.; Yaseen, S. H.; Lashine, E. S. M.; El-Fayomi, H. M.; El-Sadek, M. E. Eur. J. Med. Chem., 2007, 42, 152-160.

18. Sahu, J. K.; Ganguly, S.; Kaushik. A. J. Adv. Pharm. Tech. Res., 2014, 5(2), 90-95.

19. Karegoudar, P.; Prasad, D. J.; Ashok, M.; Mahalinga, M.; Poojary, B.; Holla, B. S. Eur. J. Med. Chem., 2008, 43(4), 808-815.

20. Ramaprasad, G. C.; Kalluraya, B.; Kumar, B. S.; Mallya, S. Der Pharma Chemica., 2012, 4(3), 1026-1032.

21. Sunil, D.; Isloor, A. M.; Shetty, P. Der Pharma Chemica., 2009, 1(2), 19-26.

22. Husain, A.; Naseer, M. A.; Sarafroz, M. Acta Polo. Pharm. Drug Res., 2009, 66, 135-140.

23. Krall, R. J.; Penry, J. K.; White, B. G.; Kupferberg, H. J.; Swinyard, E. A. Epi., 1978, 19(4), 409-428.

24. Porter, R. J.; Cereghino, J. J.; Gladding, G. D.; Hessie, B. J.; Kupferburg, H. J.; Scoville, B. Clev. Cli., 1984, 51, 293-305.

25. Lien, E. J.; Liuo, R. C. H.; Shinoucla, H. G. J. Pharm. Sci., 1979, 68, 463-468.

26. Farrar, V. A.; Ciechanowicz-Rutkowska, M.; Grochowski, J.; Serda, P.; Pilati, T.; Filippini, G.; Hinko, C. N.; El-Assadi, A.; Moore, J. A.; Edafiogho, I. O.; Andrews, C. W.; Cory, M.; Nicholson, J. M.; Scott, K. R. J. Med. Chem., 1993, 36, 3517-3525. 